\title{
Who does what in the microbiome?
}

Recent studies have shown that the complement of gut bacteria varies among individuals, but specific data linking the bacteria present to their functions in human physiology have been lacking. In a recent report, Min Li and colleagues describe a multidisciplinary approach to link the functions of the trillions of microbial gut bacteria - the human microbiome - to host metabolic phenotypes. Importantly, they pinpoint specific microorganisms that have defined roles in human metabolism using a functional genomics approach.

A four-generation Chinese family volunteered for the study. Faeces and urine were sampled on two separate occasions and analysed by molecular and spectroscopic techniques. Then, multivariate data analysis was used to identify relationships between individual bacterial species and metabolites. Sequencing of a faecal clone library revealed that all seven adult family members had gut microbiomes that were dominated by Firmicutes and Bacteroidetes, which is consistent with previous studies. Information from the metagenomic population survey was used to select primers specific for members of the microbiota. The different compositions of bacterial strains that were present in different family members were then analysed by denaturing gradient gel electrophoresis (DGGE).

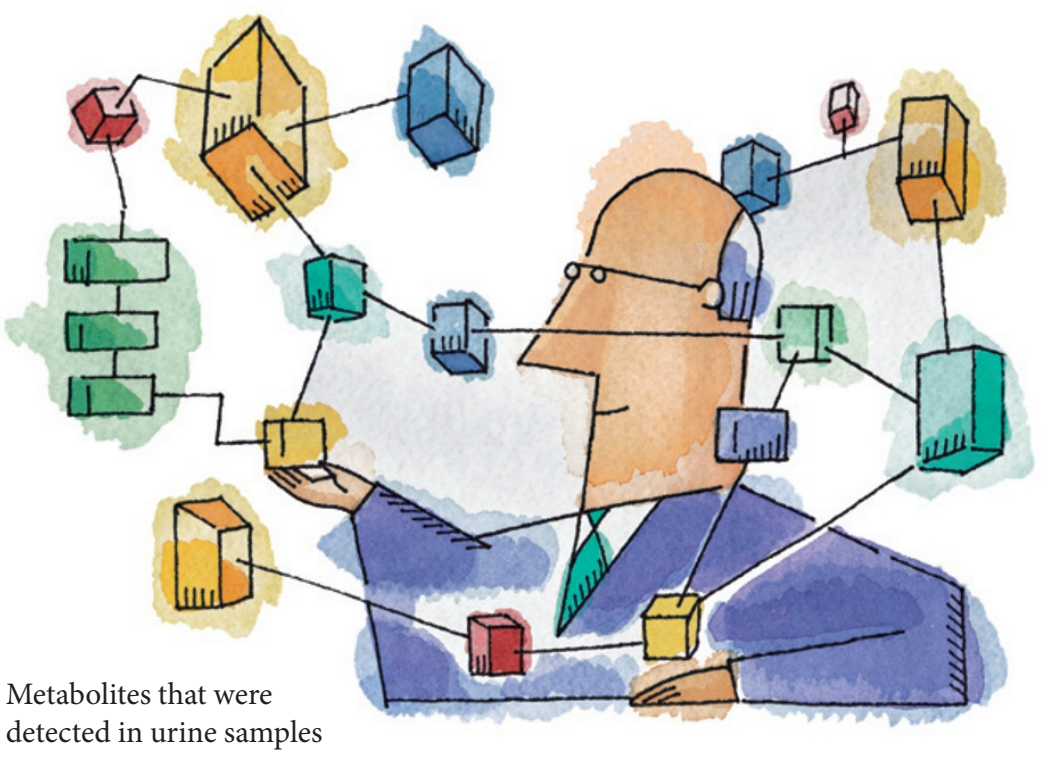

by NMR spectroscopy were linked to the DGGE fingerprint using multivariate analysis. By way of proof of principle, Li et al. linked the well-characterized species Faecalibacterium prausnitzii with 3aminoisobutyrate, one of its known metabolites. The production of 20 metabolites was linked to individual bacteria; some bacteria were only linked to one metabolite, whereas others were linked to several, which indicates that co-metabolism of food by humans and microorganisms is complex.

The metagenomic analysis was too variable and qualitative to include in the multivariate analysis, but the authors point out that sequence-based analyses should eventually become quantitative, and so, in the future, individual genes could be associated with particular metabolites. This new approach should enable the identification of those bacteria that have the biggest influence on human metabolism and are therefore the most interesting to study in terms of human health.

\section{Susan Jones}

ORIGINAL RESEARCH PAPER Li, M. et al. Symbiotic gut microbes modulate human metabolic phenotypes. Proc. Natl Acad. Sci USA 105, 2117-2122 (2008) 